\title{
Estudo da eficácia de espuma para a depopulação de aves para situações de emergência sanitária
}

\author{
Experimental evaluation of foam-based poultry depopulation for emergencies
}

\author{
Masaio Mizuno ISHIZUKA루 Walter Kazuhiko ISHIZUKA ${ }^{2}$; Fernando Gomes BUCHALA ${ }^{3}$; Ricardo de \\ ALBUQUERQUE ${ }^{4}$; Eliana Reiko MATUSHIMA ${ }^{5}$; Raphael Lúcio ANDREATTI FILHO ${ }^{6}$ \\ ${ }^{1}$ Departamento de Medicina Veterinária Preventiva e Saúde Animal da Faculdade de Medicina Veterinária e Zootecnia da \\ Universidade de São Paulo, São Paulo-SP, Brasil \\ ${ }^{2}$ Masaio Assessoria de Informações Científicas Ltda, São Paulo-SP, Brasil \\ ${ }^{3}$ Coordenadoria de Defesa Agropecuária da Secretaria de Agricultura e Abastecimento do Governo do Estado de São Paulo, \\ Campinas-SP, Brasil \\ ${ }^{4}$ Departamento de Nutrição e Produção Animal da Faculdade de Medicina Veterinária e Zootecnia da Universidade de São \\ Paulo, Pirassununga-SP, Brasil \\ ${ }^{5}$ Departamento de Patologia da Faculdade de Medicina Veterinária e Zootecnia da Universidade de São Paulo, São Paulo-SP, \\ Brasil \\ ${ }^{6}$ Departamento de Clínica Veterinária da Faculdade de Medicina Veterinária e Zootecnia da Universidade Estadual Paulista, \\ Botucatu-SP, Brasil
}

\begin{abstract}
Resumo
São inúmeras as dificuldades observadas na depopulação de elevado número de aves e é importante proceder-se à depopulação no interior do próprio aviário por questões de biossegurança do homem em casos de emergência sanitária causada por doenças de aves de elevada transmissibilidade, como a influenza aviária e a doença de Newcastle. Construiuse o equipamento gerador de espuma para provocar a anóxia física em aves. Verificou-se a eficácia da espuma gerada através do uso de líquido detergente de alta expansão sobre o sacrifício de galinhas, avaliando-se os sinais sensoriais, tempo de morte, letalidade e lesões macro e microscópicas. Duzentas galinhas de postura com 78 semanas de idade foram divididas em dez grupos experimentais e submetidas a diferentes períodos de exposição à espuma, variando de $5 \min 30$ s a $8 \min 15 \mathrm{~s}$. A partir de seis minutos de exposição à espuma $100 \%$ das aves foram mortas. A presença de bolhas de espuma na cavidade oral e traqueia foram constatadas em todos os períodos de exposição à espuma, indicando a ocorrência de asfixia física. Congestão nas mucosas da cavidade oral e traqueia foram também observadas em todos os períodos de exposição. No exame histopatológico dos pulmões de todas as aves, observou-se a presença de congestão e hemorragia difusa de moderada intensidade. Nenhuma alteração foi observada na traqueia das aves. A eficácia da espuma como método de depopulação de aves foi constatada neste trabalho, podendo ser recomendada diante da necessidade de atendimento a emergências sanitárias.
\end{abstract}

Palavras-chave: Influenza aviária. Depopulação. Espuma. Hipóxia mecânica. Aves.

\begin{abstract}
There are several difficulties in the control of quick spreading and highly contagious poultry diseases such as avian influenza and Newcastle disease, which require large numbers of poultry to be rapidly killed inside the poultry houses in order to minimize human exposure and disease spreading. We have built and tested an equipment that uses a waterbased foam from high expansion fire detergent that induces poultry anoxia measured by sensorial signs, time to death, lethality and macro and microscopically lesions. Two hundred 78-weeks old hens were divided into ten groups and exposed to different times of death according to exposure to water-foam varying from $5 \mathrm{~min} 30 \mathrm{~s}$ to $8 \mathrm{~min} 15 \mathrm{~s}$. All poultry died after six minutes of water-foam cover. Foam bubbles were observed in oral cavity and trachea of all poultry at each time of exposure to foam, revealing physical asphyxia. Macroscopically, congestion in oral cavity and in trachea mucosa was observed. Histopathology revealed diffuse and moderate hemorrhage in lungs and no lesion in trachea. The efficacy of water-foam procedure for poultry depopulation was demonstrated validating its recommendation in case of emergency.
\end{abstract}

Keywords: Avian influenza. Depopulation. Foam. Mechanical hypoxia. Poultry. 


\section{Introdução}

Doenças como a influenza aviária (IA) altamente patogênica e compondo a lista A do código zoosanitário internacional da Organização Mundial de Saúde Animal (OIE/OMSA) determinam, além do impacto econômico aos países onde ocorre a doença, sérias preocupações quanto ao método de sacrifício e destino dos animais acometidos, pois em se tratando de avicultura, geralmente há o envolvimento de milhares de aves simultaneamente. A IA é doença infecciosa, altamente contagiosa das aves causada pelo vírus do tipo A da influenza e que acomete aves domésticas e silvestres podendo manifestar ou não sinais clínicos da doença, que podem ser desde um quadro leve com comprometimento da produtividade até um quadro agudo e grave envolvendo os sistemas respiratório, nervoso e digestivo com consequente morte das aves ${ }^{1,2}$.

As estirpes patogênicas para aves possuem hemaglutinina H5 ou H7 e em 1997, o subtipo H5N1, também reconhecido como de alta patogenicidade (AP), revelou ser letal para aves domésticas e de vida livre, bem como de infectividade e patogenicidade em humanos com consequências sempre fatais. Não há nenhum caso de influenza humana registrada que tenha sido adquirida de aves silvestres ${ }^{3}$, embora patos selvagens, cegonhas e outras aves migratórias tenham sérias implicações como reservatórios do vírus $\mathrm{H} 5 \mathrm{~N}^{4}$. A capacidade de mutação do vírus da IA está diretamente relacionada a diversos surtos da doença em aves domésticas e silvestres, pequenos mamíferos, mamíferos marinhos, suínos, equinos e humanos e sua emergência deve-se muito mais ao aumento da população avícola, hábitos alimentares, limitação econômica e demográfica, negligência da vigilância e tardia notificação dos primeiros casos e menos influenciada pelas condições climáticas ${ }^{5}$.

Segundo a OIE ${ }^{1}$, como o vírus da IA de AP tem sido isolado de galinhas e perus, em casos de surtos recomenda depopulação de todas as aves afetadas e dos comunicantes. A FAO foi incumbida pela OIE em estabelecer as diretrizes para a prevenção e controle da doença para países infectados e indenes ${ }^{6}$. O Manual for Countries at Risk Preparing for Highly Pathogenic Avian Influenza preparado pela OIE-FAO-OMS/ WHO, descreve que a erradicação da IA fundamenta-se, dentre as inúmeras medidas de prevenção, na eliminação de todas as aves infectadas e as potencialmente infectadas (comunicantes) com destruição das carcaças, objetivando prevenir a ocorrência de novos casos principalmente naqueles locais onde existe elevado risco de infecção humana ${ }^{6}$.

A OIE ${ }^{7}$ estabelece os aspectos que devem nortear os procedimentos de eliminação de aves como rapidez na execução com minimização do manuseio e movimentação das aves; conduzir à morte imediata ou à perda da consciência até que ocorra a morte sem causar aversão, ansiedade, dor, aflição ou sofrimento; prever sacrifício nas próprias instalações de criação; analise do custo do processo, tipo do alojamento, de criação, localização e disponibilidade de equipamentos; considerar aspectos de segurança ambiental e das pessoas envolvidas no sacrifício; e adequada seleção do método de sacrifício. Sugerem para aves de todas as idades a aplicação de $\mathrm{CO}_{2}$ misturado ao ar ou nitrogênio e/ou gás inerte; uso de pistola sem penetração, eletrocussão pela imersão das aves em água, atordoamento elétrico; ou adição de barbitúricos ou outra droga na água ou ração.

O sacrifício humanitário objetiva proteger a saúde pública, eliminar animais fontes de infecção e comunicantes para proteger os susceptíveis, manter a saúde dos plantéis avícolas de uma nação e manter ou restaurar o status de país livre de doença, com finalidade de preservar a competitividade e recomendada quando da ocorrência de surtos ou epidemias. Concluem que o emprego de métodos que causam anóxia é mais humano do que misturas que contenham $\mathrm{CO}_{2}$, em mistura ou não com gases anóxicos como nitrogênio e argônio; que a decapitação e $\mathrm{CO}_{2}$ causam dor e aflição 
e o atordoamento com $\mathrm{CO}_{2}$ ou elétrico pode comprometer as intenções humanitárias de eliminação da dor e sofrimento ${ }^{8,9}$. O surto de IA na Holanda em 2003 com eliminação de 30 milhões de aves em um raio de $3 \mathrm{~km}$ permitiu avaliar os métodos de depopulação disponíveis à época, tendo sido obtido melhor resultado com uso de gás na própria instalação quando comparado ao sistema móvel que determinava estresse e eventual dor pelas injúrias decorrentes da captura e manipulação manual de aves vivas para colocação no equipamento móvel ${ }^{10}$.

No Brasil, o Ministério da Agricultura, Pecuária e Abastecimento (MAPA), por meio do Plano Nacional de Sanidade Avícola (PNSA), elaborou o Plano de Contingência para Influenza Aviária e Doença de Newcasthe (abril de 2007) ${ }^{11}$ para atualizar e harmonizar normas e procedimentos para a prevenção e controle destas doenças no território brasileiro, tendo como referência as recomendações da $\mathrm{OIE}^{7}$. Em caso de foco recomenda seleção de método de sacrifício imediato de todas as aves existentes e destaca que o método de despovoamento por espuma encontra-se em fase de teste ${ }^{11}$.

De acordo com a Resolução No 714 de 20/6/2002 do Conselho Federal de Medicina Veterinária do Brasil $^{12}$, eutanásia é o meio de eliminar a dor e o distresse; o sofrimento dos animais que não podem ser eliminados por meio de analgésicos, sedativos ou outros tratamentos; e quando o animal constituir ameaça à saúde pública ou animal ou for objeto de ensino ou pesquisa. Segundo a Associação Americana de Medicina Veterinária (AVMA) ${ }^{13,14}$, eutanásia é o método usado para causar a morte de um animal de forma indolor e com o mínimo de estresse e, depopulação é a destruição de grande quantidade de animais de forma rápida e eficiente considerando o bem-estar animal e as circunstâncias extenuantes do processo, e aprova o método inédito que utiliza espuma gerada a partir de solução aquosa de detergente para depopulação em massa de aves quando da ocorrência de doenças alta- mente transmissíveis de acordo com os procedimentos delineados pelo Departamento de Agricultura dos Estados Unidos da América e o Serviço de Inspeção de Saúde Vegetal e Animal (USDA/APHIS). O Comitê de Doenças Transmissíveis de Aves Domésticas e outras espécies de aves da USDA/APHIS ${ }^{14}$ emitiu parecer favorável ao uso de espuma por meio da Resolução No 44 de 18/12/2006. Dentre os itens mencionados, estabelece que $95 \%$ das aves devem morrer em até sete minutos a contar da completa submersão e que as remanescentes após 15 minutos devem ser sacrificadas rápida e humanitariamente.

O método de depopulação de aves baseado no princípio da espuma utilizada em incêndios foi descrito por Dawson et al ${ }^{15}$ diante da dificuldade em se utilizar o tradicional sacrifício pelo uso de $\mathrm{CO}_{2}$ que, a despeito de ser humanitário, exige condições especiais de vedação das instalações, elevado número de pessoas e quando do envolvimento de zoonose, requer medida adicional de proteção humana. Estudo do método da espuma comparativamente ao $\mathrm{CO}_{2}$ e da mistura de ambos revelou que a espuma é aplicável em plantéis com elevado número de aves e provocando menos estresse quando avaliada pela dosagem de níveis de corticosteróide ${ }^{16}$.

Diante das dificuldades descritas na literatura para depopulação de elevado número de aves, construiu-se equipamento gerador de espuma capaz de determinar anóxia física em aves para utilização em situações de emergência sanitária causada por doenças de aves de elevada transmissibilidade como IA e doença de Newcastle. Assim, o objetivo do presente experimento foi a de avaliar a eficácia de espuma gerada por líquido de alta expansão no sacrifício de galinhas mensurando os sinais sensoriais, tempo de morte, letalidade e lesões macro e microscópicas.

\section{Material e Método}

Foram utilizadas 200 galinhas adultas da linhagem Hy Line Brown com 78 semanas de idade, provenien- 
tes de granja comercial e aleatoriamente selecionadas dentre as demais componentes do plantel avícola. As aves foram conduzidas ao aviário do Departamento de Produção e Nutrição Animal da Faculdade de Medicina Veterinária e Zootecnia da Universidade de São Paulo - Campus Pirassununga, mantidas em repouso e em jejum até o momento do experimento, em período não superior a seis horas.

O equipamento para produção de espuma constituiu-se de bomba de água do tipo centrífuga com alta capacidade de vazão e acionada a gasolina e/ou eletricidade; sistema de injeção do líquido gerador de espuma no fluxo principal de água por meio de bomba dosadora com regulagem de vazão; carretel para sustentação da mangueira durante a operação sob pressão; dispositivo de formação de espuma acoplado na extremidade final da mangueira e quadro de comando elétrico. Utilizou-se o produto JET-X - High Expansion Foam Concentrate (ANSUL Incorporated, USA) como líquido gerador de espuma. Para produção de espuma houve regulagem da vazão desejada por meio de moto bomba de recalque misturada ao líquido gerador de espuma na proporção de $2 \%$ recalcada até o dispositivo gerador de espuma para sucção do ar e formação de bolhas de espuma com diâmetro médio de $5 \mathrm{~mm}$. O equipamento foi projetado e construído para produzir cerca de $20 \mathrm{~m}^{3}$ de espuma por minuto, ou seja, $1.200 \mathrm{~m}^{3}$ de espuma/hora.

O aviário foi fechado e utilizando-se de placas flexíveis de madeira, construiu-se local para contenção das aves com capacidade para 30 galinhas adultas. As aves foram submetidas em tempos distintos e sucessivos de imersão em espuma divididos em dez grupos experimentais de 20 galinhas respectivamente para $5 \min 30 \mathrm{~s}, 5 \min 45 \mathrm{~s}, 6 \mathrm{~min}, 6 \min 45 \mathrm{~s}, 7 \mathrm{~min}$, $7 \min 15 \mathrm{~s}, 7 \min 30 \mathrm{~s}, 7 \min 45 \mathrm{~s}, 8 \min$ e $8 \min 15 \mathrm{~s}$. A espuma foi aplicada sobre o piso cobrindo paulatinamente as aves e para assegurar a submersão total, aplicou-se a espuma até que esta atingisse $20 \mathrm{~cm}$ acima da altura máxima das galinhas. Cada grupo experimental foi submetido ao tempo estabelecido de imersão, quantificado o número de aves mortas, que foram imediatamente submetidas à necropsia para exame anatomopatológico, e seis foram aleatoriamente selecionadas para colheita de amostras de pulmão e traqueia para exame histopatológico, utilizando-se coloração pelo método de Hematoxilina Eosina de cortes de $5 \mathrm{~mm}$ preparados a partir de fragmentos dos órgãos previamente fixados em formol a $10 \%$ por 24 horas, desidratados, diafanizados e incluídos em parafina. As lâminas foram preparadas no Laboratório de Histologia do Departamento de Patologia da Faculdade de Medicina Veterinária e Zootecnia da Universidade de São Paulo e submetidas à leitura em microscopia de luz com aumentos de 40x, 100x, 400x e 1000x.

O método estatístico constitui-se na aplicação do teste de duas proporções com aproximação Normal ${ }^{17}$ e o valor de $\alpha$ (alfa) foi fixado em 0,05.

\section{Resultados e Discussão}

Os resultados foram baseados nos critérios de avaliação dos sinais sensoriais e morte das aves, além das lesões macro e microscópicas. A avaliação da morte foi realizada contando-se o tempo (minutos) a partir da submersão total das galinhas na espuma. A constatação da morte foi verificada pelos sinais sensoriais das aves, baseando-se na Instrução Normativa $N^{0} 3$ de 17/01/2000 do BRASIL ${ }^{18}$ que estabelece em aves insensibilizadas em abatedouros os sinais sensoriais representados por asas junto ao corpo, pescoço distendido, bico sem movimento e ausência de reflexo ocular. Procedimento padrão de necropsia em aves domésticas foi adotado para exame dos sistemas respiratório (laringe, traqueia e sacos aéreos) e digestivo (cavidade oral), com particular atenção à presença de bolhas de ar, congestão e hemorragia, seguindo-se o exame histopatológico dos fragmentos de pulmões e traqueia. 
Na tabela 1 está demonstrada a letalidade de galinhas submetidas ao método de espuma em relação ao tempo de exposição. Considerando os sucessivos tempos de exposição à espuma, observou-se que a letalidade de 80,0\% aos $5 \mathrm{~min} 30$ s e de $70,0 \%$ aos $5 \mathrm{~min} 45 \mathrm{~s}$ foram estatisticamente iguais e inferiores à letalidade de 100,0\% observada a partir de 6 min até o final do experimento que foi de $8 \mathrm{~min} 15 \mathrm{~s}$. Este valor não se afasta da observação de Benson et al. ${ }^{16}$ que, trabalhando com frangos de corte monitorados por meio de eletrocardiograma, observaram que o método de espuma provocou a morte das aves em 4 min57s. No entanto, diferem das observações de Dawson et al. ${ }^{15}$ que realizaram experimento com frangos de corte comparando uso de $\mathrm{CO}_{2}$ associado ou não à espuma e a espuma isoladamente como métodos para depopulação de aves. $\mathrm{O}$ uso somente de $\mathrm{CO}_{2}$ provocou a morte das aves em 2:08 min, a associação $\mathrm{CO}_{2}$ e espuma em 2 min 09 s e apenas a espuma em 2 min54s. Estes mesmos autores observaram que, durante o processo de sacrifício, as aves submetidas ao $\mathrm{CO}_{2}$ associado ou não à espuma apresentaram visível agitação, fato não observado quando da utilização isolada da espuma, cujas aves permaneceram calmas e sem sinal aparente de estresse.

As proporções de aves mortas e necropsiadas com presença de bolhas de espuma observadas na cavida- de oral e nas porções anterior, média e posterior da traqueia variaram de $85,0 \%$ a $100,0 \%$ tendo sido estatisticamente iguais em todos os tempos de exposição à espuma. Estes resultados permitem inferir que a presença de bolhas de espuma na cavidade oral e na traqueia são fatores importantes para determinar a asfixia física das aves.

A presença de congestão da mucosa da cavidade oral e nas porções anterior, média e posterior da traqueia de galinhas mortas após terem sido submetidas ao método de espuma e necropsiadas foi observada na totalidade das aves a partir de seis minutos de exposição. No exame histopatológico dos pulmões e traqueia, observou-se a presença de congestão e hemorragia difusa de moderada intensidade nos pulmões e nenhuma alteração foi observada nas traqueias de todas as aves examinadas. Dawson et al. ${ }^{15}$ e Benson et al. ${ }^{16}$ observaram a presença de hemácias na traqueia, siringe e pulmões de aves sacrificadas por espuma ou $\mathrm{CO}_{2}$. Além destes métodos de depopulação, o sacrifício de aves por meio do deslocamento cervical, também determina o acúmulo de sangue no sistema respiratório. Os resultados observados por Dawson et al. ${ }^{15}$ e Benson et al. ${ }^{16}$, bem como os deste trabalho, indicam que as aves sacrificadas por espuma morrem

Tabela 1 - Mortalidade de galinhas sacrificadas pelo método de espuma em relação ao tempo de exposição - São Paulo - 2008

\begin{tabular}{cccc}
\hline $\begin{array}{c}\text { Tempo de exposição } \\
\text { (minutos) }\end{array}$ & $\begin{array}{c}\text { Quantidade de aves mortas / } \\
\text { Quantidade total de aves }\end{array}$ & Letalidade (\%) & $\begin{array}{c}\text { Sinais sensoriais nas } \\
\text { sobreviventes }\end{array}$ \\
\hline $5: 30$ & $16 / 20$ & $80,0^{\mathrm{b}}$ & Todos os sinais presentes \\
$5: 45$ & $14 / 20$ & $70,0^{\mathrm{b}}$ & Todos os sinais presentes \\
$6: 00$ & $20 / 20$ & $100,0^{\mathrm{a}}$ & \\
$6: 45$ & $20 / 20$ & $100,0^{\mathrm{a}}$ & \\
$7: 00$ & $20 / 20$ & $100,0^{\mathrm{a}}$ & \\
$7: 15$ & $20 / 20$ & $100,0^{\mathrm{a}}$ & \\
$7: 30$ & $20 / 20$ & $100,0^{\mathrm{a}}$ & \\
$7: 45$ & $20 / 20$ & $100,0^{\mathrm{a}}$ & \\
$8: 00$ & $20 / 20$ & $100,0^{\mathrm{a}}$ & \\
$8: 15$ & $20 / 20$ & $100,0^{\mathrm{a}}$ & \\
\hline
\end{tabular}

Para letras em sobrescrito diferentes têm-se que $a>b$. 
por hipóxia, não havendo nenhuma evidência de que a morte tenha ocorrido por afogamento.

Tendo sido o equipamento projetado para produzir cerca de $20 \mathrm{~m}^{3}$ de espuma por minuto, ou seja, 1.200 $\mathrm{m}^{3}$ de espuma/hora, o uso em galpão comercial medindo $12,0 \mathrm{~m} \mathrm{x} \mathrm{100,0} \mathrm{m}\left(1.200 \mathrm{~m}^{2}\right)$ e admitindo a necessidade de 0,90 $\mathrm{m}$ de altura de espuma, o espaço de $1.080 \mathrm{~m}^{3}$ pode ser preenchido em $54 \mathrm{~min}$.

\section{Conclusão}

A eficácia da espuma como método de depopulação de aves foi constatada neste trabalho, podendo ser recomendada diante da necessidade de aten-

\section{Referências}

1. OIE. Animal disease data: highly pathogenic avian influenza. 2002. Disponível em: <http://www.oie.int/eng/maladies/en_ technical_diseasecards.htm>. Acesso em: 20 dez. 2006.

2. KALETA, E. F.; HERGARTEN, G.; YILMAZ, A. Slaughter of poultry during the epidemic of avian influenza in the Netherlands in 2003. Dtsch Tierarztl Wochenschr, v. 112, n. 12, p. 448-456, 2005.

3. SWAYNE, D. E.; HALVORSON, D. A. Influenza. In: SAIF, Y. M. Diseases of poultry. 11. ed. Iowa: Iowa State Press, 2003. $1231 \mathrm{p}$.

4. DUDLEY, J. P. Wildlife and human implications of emerging viral zoonotic diseases in southeast Asia. Recenti Progressi in Medicine, v. 96, n. 11, p. 523-534, 2005.

5. CHASTEL, C. Emergence of new viruses in Asia: is climate change involved? Medecine et Maladies Infectieuses, v. 11, n. 34, p. 499-505, 2004.

6. MARTIN, V.; FORMAN, A.; LUBROTH, J. Preparing for highly pathogenic avian influenza. In: countries at risk. Roma: FAO \& OIE, 2006.

A Manual for

7. OIE. Guidelines for the killing of animals for diseases control purposes. In: OIE, 2006. cap. 3.7.6.

8. RAJ, M. Welfare, economic and practical implications of gas stunning prior to poultry slaughter. In: SEMINAR US DEPARTMENT OF AGRICULTURE, 2004, Washington DC. 2004.

9. RAJ, M. Caring during crisis: animal welfare during pandemics and natural disasters. In: HUMANE KILLING OF ANIMALS FOR DISEASE CONTROL PURPOSES, 2007, Guelph. Proceedings... Guelph, Canada: University Guelph, 2007.

10.GERRITZEN, M. A.; LAMBOOIJ, E.; STEGEMAN, J. A.; SPRUIJT, B. M. Slaughter of poultry during the epidemic of dimento a emergências decorrentes de IA e outras doenças altamente contagiosas. Os parâmetros avaliados permitem dizer que a utilização da espuma determina índices compatíveis com os recomendados por entidades internacionais que regulamentam procedimentos desta natureza, além de permitir a depopulação de elevada quantidade de aves e a sua realização no interior dos aviários, satisfazendo assim os aspectos de biossegurança. O método apresenta a vantagem de permitir o dimensionamento do volume de espuma necessário de acordo com a quantidade aves a serem eliminadas e a área onde se encontram alojadas.

avian influenza in the Netherlands in 2003. The Veterinary Record, v. 159, n. 2, p. 39-42, 2006.

11.BRASIL. Ministério da Agricultura, Pecuária e Abastecimento. Plano Nacional de Contingência para Influenza Aviária e Doença de Newcastle. Brasília: MAPA, 2007. (Versão 1.2).

12.CONSELHO FEDERAL DE MEDICINA VETERINÁRIA. CFMV. Procedimentos e métodos de eutanásia em animais. Resolução No 714 de 20/6/2002. Disponível em: <http://www. protetoresvoluntarios.com.br/modules/smartsection/item. php?itemid=1>. Acesso em: 10 set. 2006.

13.2000 Report of the AVMA Panel on euthanasia. Journal American Veterinary Medical Association, v. 218, n. 5, p. 669-696, 2001

14.AMERICAN VETERINARY MEDICAL ASSOCIATION. AVMA. Use of water-based foam for depopulation of poultry. 2006. Disponível em: <www.avma.org/issues/policy/ poultry_depopulation.asp>. Acesso em: 01 dez. 2007.

15.DAWSON, M. D.; BENSON, E. R.; MALONE, G. W.; ALPHIN, R. L.; ESTEVEZ, I.; VAN WICKLEN G. L. Evaluation of foam-based mass depopulation methodology for floor-reared meat-type poultry operations. Applied Engineering Agriculture, v. 22, n. 5, p. 787-793, 2006.

16.BENSON, E.; MALONE, G. W.; ALPHIN, R. L.; DAWSON, M. D.; POPE, C. R.; VAN WICKLEN, G. L. Foam-based mass emergency depopulation of floor-reared meat-type poultry operations. Poultry Science, v. 86, n. 2, p. 219-224, 2007.

17.VIEIRA, S. Introdução à bioestatística. Rio de Janeiro: Campus, $1981.294 \mathrm{p}$.

18. BRASIL. Ministério da Agricultura, Pecuária e Abastecimento. Instrução Normativa n. 3 de 17 de janeiro de 2000. Regulamento Técnico de métodos de insensibilização para o abate humanitário de animais de açougue. Brasília: MAPA, 2000 . 